\title{
Performance and flow dynamics studies of polymeric optofluidic SERS sensors
}

\author{
S. Uusitalo \\ Sanna.uusitalo@vtt.fi
}

\section{J. Hiltunen}

\section{P. Karioja}

S. Siitonen

\section{Kontturi}

R. Myllylä

\section{Kinnunen}

\section{Meglinski}

\author{
VTT Technical Research Centre of Finland, Kaitoväylä 1, 90590 Oulu, Finland \\ University of Oulu, Pentti Kaiteran katu 1, 90014 Oulu, Finland \\ VTT Technical Research Centre of Finland, Kaitoväylä 1, 90590 Oulu, Finland \\ VTT Technical Research Centre of Finland, Kaitoväylä 1, 90590 Oulu, Finland
}

Nanocomp Oy Ltd, Ensolantie 6, 80710 Lehmo, Finland

Nanocomp Oy Ltd, Ensolantie 6, 80710 Lehmo, Finland

University of Oulu, Pentti Kaiteran katu 1, 90014 Oulu, Finland

University of Oulu, Pentti Kaiteran katu 1,90014 Oulu, Finland

University of Oulu, Pentti Kaiteran katu 1, 90014 Oulu, Finland

We present a polymer-based optofluidic surface enhanced Raman scattering chip for biomolecule detection, serving as a disposable sensor choice with cost-effective production. The SERS substrate is fabricated by using industrial roll-to-roll UV-nanoimprinting equipment and integrated with adhesive-based polymeric microfluidics. The functioning of the SERS detection on-chip is confirmed and the effect of the polymer lid on the obtainable Raman spectra is analysed. Rhodamine 6C is used as a model analyte to demonstrate continuous flow measurements on a planar SERS substrate in a microchannel. The relation between the temporal response of the sensors and sample flow dynamics is studied with varied flow velocities, using SERS and fluorescence detection. The response time of the surface-dependent SERS signal is longer than the response time of the fluorescence signal of the bulk flow. This observation revealed the effect of convection on the temporal SERS responses at $25 \mu \mathrm{l} / \mathrm{min}$ to $1000 \mu \mathrm{l} / \mathrm{min}$ flow velocities. The diffusion of analyte molecules from the bulk concentration into the sensing surface induces about a 40-second lag time in the SERS detection. This lag time, and its rising trend with slower flow velocities, has to be taken into account in future trials of the optofluidic SERS sensor, with active analyte binding on the sensing surface.

[DOI: http://dx.doi.org/10.2971/jeos.2015.15043]

Keywords: SERS optofluidic polymer biosensor continuous flow

\section{INTRODUCTION}

Optical biosensing is a diverse and evolving research field aiming for simplified and high-sensitivity diagnostic tools for the detection of biologicals/chemicals in environmental monitoring, medical analysis, food safety, and security. In particular, label-free approaches are interesting since they do not require label molecules to be added, which could disturb the binding event, non-specifically adsorb to the surface and complicate the process with extra cleaning steps. This has motivated the development of label-free optical sensor technologies, including interferometric, resonant-cavity, photonic crystal, surface plasmon resonance (SPR), and surface-enhanced Raman scattering (SERS) sensors [1]-[3].

Polymer materials are particularly attractive in optical sensing because of their ability to be processed rapidly and costeffectively with high yields. Polymers attain a large number of good optical properties, including high optical transmit- tance, versatile processability at relatively low temperatures, and the potential for low-cost mass-production. UV lithography has been widely used in the fabrication of conventional optical devices. The resolution obtained with this technique is limited by the effects of wave diffraction and scattering. Compared with conventional techniques, UV-imprint lithography is easy to perform, requires low-cost equipment, and can provide high-resolution nano-scale features down to sub$10 \mathrm{~nm}$. UV-imprint lithography is performed by pressing a mould onto a UV-sensitive precursor resin coating on a substrate, and by curing under UV light, a replica of the mould is formed. The process takes place at room temperature and does not require high pressure during the imprinting process. Quite recently, new fabrication methods have been developed for the UV-imprinting of optical nanophotonic sensor structures with industrial roll-to-roll devices [4]. 
The integration of polymeric optical sensor structures with microfluidic sample-handling circuits enables the fabrication of fully polymer-based optofluidic biosensors. In optofluidic sensors with planar sensing surfaces [5], the detection of analytes from the bulk sample relies on the transport of the analytes to the detection area and to the detection surface. Since the concentration of the analytes in the sample solution is typically low and the diffusion of the molecules is slow, the used detection method has to be sensitive in order to detect and quantify the molecules. SERS is an attractive method for the detection to meet these requirements. It does not only have high detection sensitivity, in some cases even in the range of a few molecules [6]-[9], but it also has an ability to identify the analytes through specific Raman spectra. The Raman spectra consist of the inelastically scattered light of the medium under inspection. The detected spectral lines act as individual features of the sample and can distinguish the sample contents. The sensitivity of the SERS detection is a result of interaction between metal substrates or colloids and the incident light. Surface plasmons of metal enhance the Raman scattering mainly through electromagnetic enhancement mechanism, but in some cases chemical enhancement due to chemical bonds between molecules and metal can also play a part in the phenomenon [10]. The enhancement factor has been proved to be even as high as $10^{10}$ for a bunch of aggregated metal colloids and commonly around $10^{6}-10^{7}$ for metal substrates. Although the enhancement induced by the colloid aggregates can be stronger than the signal strength acquired with metal substrates, the repeatability of the signal response is worse. A similar problem exists with randomly ragged metal substrates [11]. During the last decade the repeatability issue has been tackled by fabrication of more consistent planar SERS surfaces [12]-[16]. These surfaces have plasmonic elements with a defined shape, leading to a more uniform enhancement effect. Consistent solid SERS surfaces have inspired the idea of an optofluidic SERS chip, and this has raised wide research interest in the last 10 years [17].

The current aim of a disposable optofluidic sensor requires new fabrication methods and parting from the conventional fabrication of silicon-based sensors. There are a couple of recent studies focusing on low-cost approaches such as soft lithography. In the study by Liu et al., polydimethylsiloxane (PDMS) patterning was utilised in the fabrication of SERS structures with an integrated glass microfluidic circuit [18]. Lamberti et al introduced an all-PDMS-based system in which the SERS structures were fabricated by sputtering nanoparticles on PDMS [19]. However, the industrial-scale production of consistently and accurately patterned PDMS is still quite difficult.

In this work, we introduce an optofluidic sensor, which combines polymeric roll-to-roll nano-imprinted (NIL) SERS surfaces with adhesive-based microfluidic parts. According to the previous study with a benzyl mercaptan $\left(\mathrm{C}_{6} \mathrm{H}_{5} \mathrm{CH}_{2} \mathrm{SH}\right)$ test molecule on top of the R2R fabricated SERS structure without fluidics integration, the used SERS surface can provide up to $10^{7}$ a enhancement factor with good reproducibility (5\%) [4]. A SEM image of the R2R patterned SERS surface and a closeup picture of one pyramid-shaped well are shown in Figure 1.

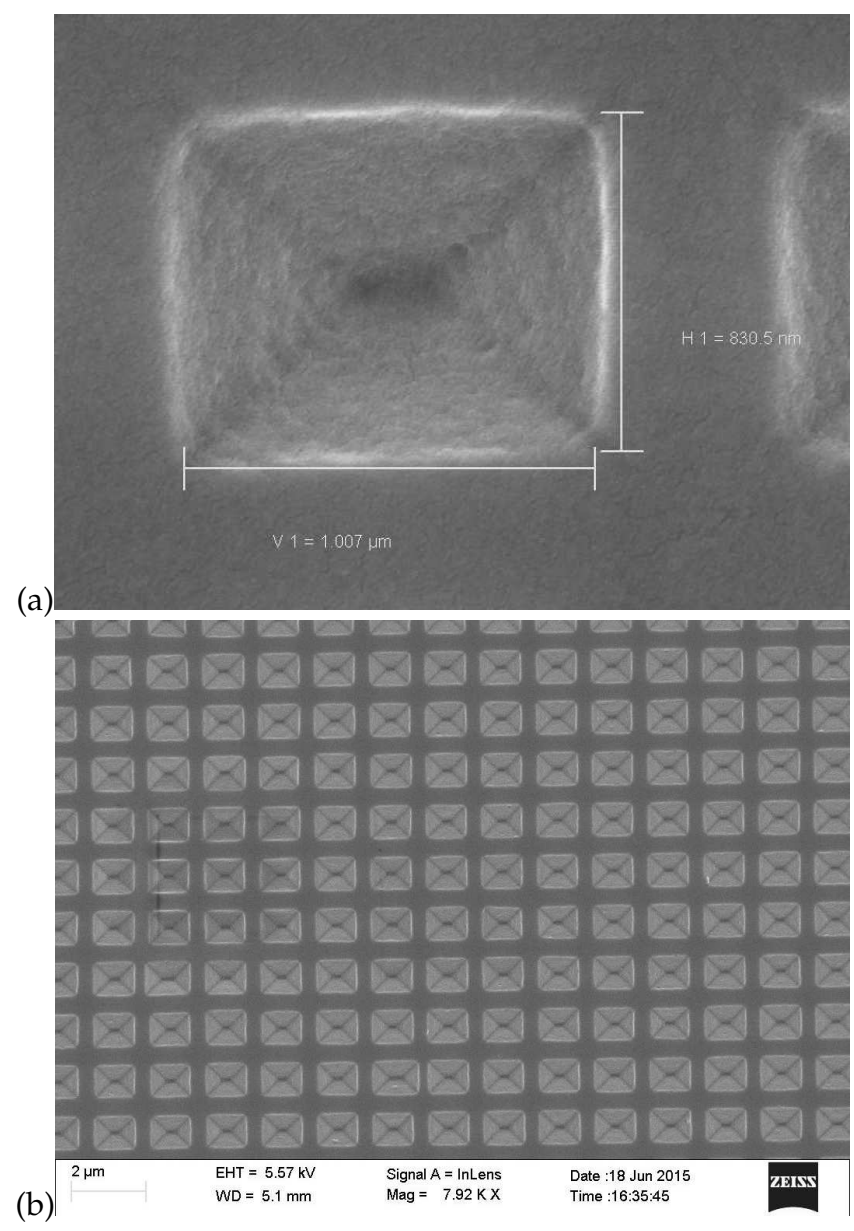

FIG. 1 a) Close-up picture of the pyramid-shaped well of the R2R fabricated SERS surface; b) A top picture of multiple SERS patterns.

The combination of a UV-imprinted SERS surface on plastic and polymer microfluidics makes the whole sensor polymerbased, with an evaporated thin gold layer as the only exception to the rule. This disposable sensor is suitable for industrial-scale fabrication with low material costs and easy fabrication methods. It enables constant monitoring of chemical and biological reactions on-chip, due to the high sensitivity of the SERS and the miniaturised sample volumes offered by the microfluidic liquid handling. Continuous flow on-chip enables consecutive sampling and washing steps, and reveals the dynamic abilities of the sensor. We studied the optical capabilities of the sensor with Rhodamine 6G (R6G) model analyte.

\section{BASIC THEORY OF FLUIDICS AND MOLECULE TRANSPORT}

To understand the effect of flow dynamics on the detected optical signal, the phenomena behind the transport of the sample molecules from the bulk flow into the detection surface must be considered. With optical detection surfaces, such as in SPR and planar SERS, the flow in a microchannel has a strong influence on the recorded signal. The bulk flow in the microfluidic channels is often produced by pump-inflicted pressure. The pressure-driven flow carries the sample molecules into the detection area, where the induced signal can be observed. In SERS, the sample molecules arriving on top of the detec- 


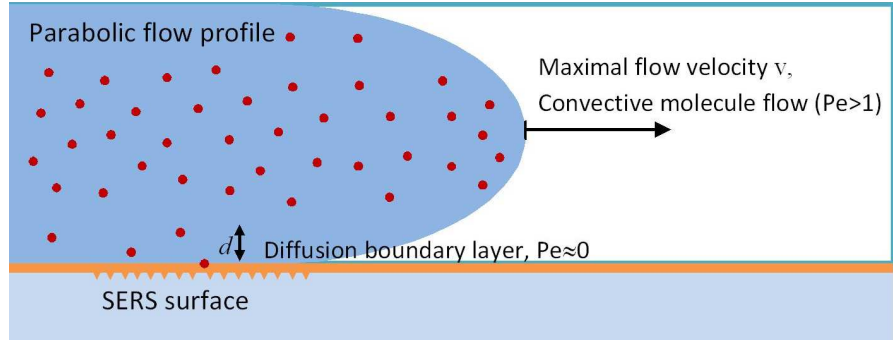

FIG. 2 A schematic of the relation between convective and diffusive flow in a microchannel. The effect of diffusion and convection can be theoretically considered through diffusion boundary layer thickness $d$ and the Peclet number. The dimensionless Peclet number is a ratio between the conductive and the diffusive transport rate of the analyte molecules. In the middle of the channel $\mathrm{Pe} \ll 1$, near the walls $\mathrm{Pe} \approx 0$ [26].

tion area need to be in the near vicinity of the plasmonic surface to be detected. Typically, the induced signal originates from the sample molecules adsorbed onto the detection surface. The nature of the flow in a microchannel can be theoretically assessed using Reynold's number. Reynold's number is a dimensionless value defining the ratio between inertial and viscous forces encountered by the fluid in the channel:

$$
R e=\rho_{m} u_{m} D_{h} / \mu_{m}
$$

where $u_{m}, \rho_{m}$ and $\mu_{m}$ are the mean flow velocity, the mean density, and the mean dynamic viscosity of the fluid, respectively. $D_{h}$ is the hydraulic diameter of the detection chamber. For a rectangular chamber, the diameter can be computed from the equation

$$
D_{h}=\frac{2 W H}{W+H},
$$

where $W$ is the width of the microchannel and $H$ is the height of the channel [20]. The flow is surely laminar when the Reynold's number is 2100 or less [21]. For a microchannel with smooth channel walls, the number is typically well under this limit. In our case the Reynolds number is 10 or less for the flow velocities used in this study, and the flow is thus laminar. The molecule transport inside a microchannel can be divided into three regions: the middle region far from channel walls, where convection (drift) is the dominant driving force of the molecules; the hybrid region, where convection is fainter and diffusion plays a role in the net effect; and the region very near the channel walls, where diffusion is the only transport mechanism. The flow profile of the pressure-driven aqueous fluid in a microfluidic channel is characteristically parabolic due to the friction between the fluid and the channel walls $[21,22]$. Thus the fluid has the highest velocity in the middle of the channel, and the velocity reduces as the observation point moves nearer to the walls. Typically the fluid velocity vanishes completely at a distance of one molecule layer from the wall. This is called the non-slip condition, in which the molecules adjacent to the channel wall do not move with the flow due to the friction between the wall and the molecules [23]-[25]. Since the flow velocity diminishes near the walls, the transport of the sample molecules inside this region by convection is negligible. Figure 2 depicts a situation where the flow velocity of the fluid is at maximum in the middle of the channel, and the convective flow vanishes near the walls.
The zone near the wall, where diffusion is the dominant transport mechanism, is called the diffusion boundary layer. There are several studies on the effect of the diffusion boundary layer on the detection mechanism of the biosensor analysed using empirical and computational methods [21, 22], [27]-[31]. The phenomena affecting the results include the binding reaction of the analytes to the sensor surface (association and dissociation rate constants) and the relation of convection and diffusion in the diffusion boundary layer [26], [32]-[35]. Studies on SPR have shown that the measured kinetic reaction rates deviate from the values acquired using other verified methods if all the phenomena related to the signal construction with planar sensing are not carefully considered. The finite element method (FEM) has been used to study the relationship between molecule transport and the adsorption process at the surface $[21,30,31]$. The optical signal response depends on the flow dynamics through the limitations of mass transport of molecules and kinetic binding reactions. In mass transport limited flow, the transport of analytes to the sensor surface is so slow that the signal rise times are growing by the lack of analyte molecules in the vicinity of the surface. This phenomenon includes the effects of insufficient molecule transport to the diffusion boundary layer by convective flow and the effect of the diffusion boundary layer. While insufficient molecule transport can cause analyte depletion near the sensor surface, the effect of the diffusion boundary layer in non-slip conditions makes the signal rise times longer due to slow molecule diffusion. As the bulk flow velocity increases, the effect of transport limitation decreases. This is due to the disappearance of the depletion effect caused by the slow convective flow. For example, kinetic reaction studies using commercial SPR sensors strive to stay outside the region where the optical response is heavily influenced by the mass transport limitation [26], [36]-[38]. By using high enough flow rates ( $\geq 50 \mu \mathrm{l} / \mathrm{min}$ ), the concentration of the analyte at the surface can be the same as that in the bulk, and the measured signal reflects binding kinetics [38]. However, with too high flow rates, the signal response can encounter a new limitation due to the reaction kinetics of the analyte binding. This kinetic limitation occurs when the binding rates are slow and bulk flow velocity is high. The analytes are transported over the detection zone so rapidly that very few of them have enough time to bind to the surface. When maximal surface coverage of analytes is desired, the used flow rate is often a compromise between the efficient transport of analyte molecules to the surface and the suitable flow velocity for adsorption [21]. Adsorption can occur with physical or chemical bonding to the surface or to the receptors/ligands on top of the surface. In this study, we can ignore the factor of analyte-ligand binding, since no ligands are at the detection surface and the bonding happens through Van der Walls forces by physisorption [39, 40]. Arrival of the analytes at the surface and the binding through physisorption can be described as a two-phase process:

$$
\begin{gathered}
A_{\text {Bulk }} \stackrel{\text { Diffusion }}{\Longleftrightarrow} A_{\text {Surface }} \\
A_{\text {Surface }} \stackrel{\text { Adsorption }}{\Longleftrightarrow} A_{\text {Bound }},
\end{gathered}
$$

where $\mathrm{A}_{B u l k}$ is the free analyte in bulk flow of liquid medium $\left[1 / \mathrm{cm}^{3}\right], A_{\text {Surface }}$ is the free analyte in liquid medium in the 


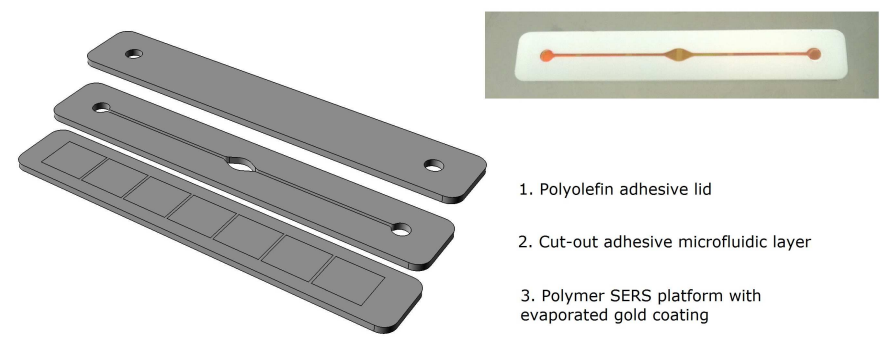

FIC. 3 Schematic of the optofluidic SERS chip with an oval detection chamber: Lid layer optically clear $3 \mathrm{M} 9795 \mathrm{R}$ polyolefin adhesive patterned with a cutting plotter, middle layer $220 \mu \mathrm{m} 3 \mathrm{M}$ adhesive patterned with the cutting plotter, and bottom layer with UV-imprinted SERS patterns and metal coating.

immediate vicinity of the surface $\left[1 / \mathrm{cm}^{3}\right]$, and $A_{\text {Bound }}$ is the adsorbed analyte on the surface $\left[1 / \mathrm{cm}^{2}\right]$.

\section{DEVICE FABRICATION}

The SERS substrates used in this study were produced by replicating the SERS pattern on top of a polymer sheet with a roll-to-roll (R2R) process. A UV-curable lacquer coating was applied on top of a PMMA carrier web using a reverse gravure technique. The SERS structure was imprinted onto the lacquer by the embossing reel, and the lacquer was cured by UV light exposure through the PMMA carrier film. The procedure is explained in detail by Oo et al. in [4]. After die-cutting separate SERS sheets from the roll, the SERS active plasmonic layer was added by evaporating a $240 \mathrm{~nm}$ gold layer on top of the polymer SERS surface. The microfluidic circuits were cut from $3 \mathrm{M}$ adhesive by cutting plotter CraftRobo Pro S. The detection chamber of the fluidic circuit had an oval shape for optimal liquid filling with a steady fluid front, and the chamber sample volume was $2 \mu \mathrm{l}$. The chamber dimensions were $220 \mu \mathrm{m}$ height and $2.67 \mathrm{~mm}$ maximal width. The channels leading into the chamber were $400 \mu \mathrm{m}$ wide. The SERS substrate and the microfluidic circuit were integrated by Yosan roll laminator at room temperature. To minimise the effect of the chamber lid on the Raman signal, the microfluidic circuit was lidded with a polyolefin diagnostic adhesive (3M 9795R), which declares high optical clarity and minimal auto-fluorescence. The picture of the SERS chip and the schematic of the layers can be seen in Figure 3.

\section{MEASUREMENT SET-UP}

Rhodamine 6G (dye content $~ 95 \%$, SigmaAldrich) solution diluted in deionized water was used as a model analyte to analyse the functioning of the polymer-based SERS chip. The optical properties of the chip were studied by filling the chip with the $\mathrm{R} 6 \mathrm{G}$ samples and $\mathrm{DI} \mathrm{H}_{2} \mathrm{O}$ serving as a reference medium for the R6G in water solutions. Water is the preferred medium to be used in Raman spectroscopy as a basis for the sample solutions and as a reference, because it does not produce Raman peaks itself. In flow trials, the chip was filled with under-pressure suction produced by a syringe pump (Nexus 3000). The sample was injected into a $2 \mathrm{ml}$ Eppendorf tube, from where it was transferred through the chip and Dolomite

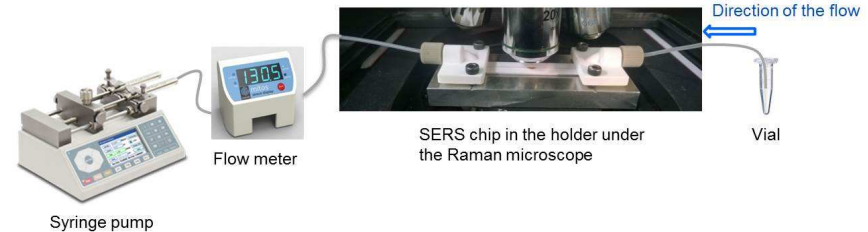

FIG. 4 The set-up for the flow studies with sample vial, chip holder, chip, Raman microscope, flow meter, and syringe pump.

flow meter into a syringe. A schematic of the flow system is presented in Figure 4.

The surface-enhanced Raman spectra were recorded using a BaySpec Nomadic Raman microscope with a 785 nm excitation wavelength. The power of the laser was set at $40 \mathrm{~mW}$ and a 20X magnifying objective was used in the experiments. Integration times were varied between $15 \mathrm{~s}$ and $30 \mathrm{~s}$ depending on the used R6G concentration. The BaySpec camera was used to focus the system by adjusting a sharp edge between the patterned SERS area and the smooth gold area through the polyolefin lid of the chamber before each Raman spectrum acquisition.

In a continuous flow study, the fluid flow velocity was varied from $25 \mu \mathrm{l} / \mathrm{min}$ to $1000 \mu \mathrm{l} / \mathrm{min}$. To separate the effect of the convective flow of molecules and the mass transport of molecules on the detection surface, we measured the flow of the bulk liquid using fluorescence microscopy and the arrival of the molecules to the detection surface with SERS. To our knowledge, this is a novel method for analysing the dynamic behaviour of an optofluidic chip. $0.5 \mathrm{mM}$ R6G was used as the model analyte. R6G fluoresces around the $570 \mathrm{~nm}$ wavelength. Water was first flowed by a syringe pump induced under pressure into the detection chamber before filling the system with dilute $0.5 \mathrm{mM}$ R6G in DI $\mathrm{H}_{2} \mathrm{O}$. The actual flow velocities were observed during the trials with a Dolomite Mitos flow sensor. The flow was recorded as avi-files using a Zeiss fluorescence microscope camera time lapse mode with a $10 \mathrm{~ms}$ exposure time and $1 \mathrm{~s}$ interval. The same flow trial was executed for SERS detection under the BaySpec Nomadic Raman microscope and the surface-enhanced Raman spectra were recorded with a $15 \mathrm{~s}$ integration time and $1 \mathrm{~s}$ interval. The results were analysed using Matlab and Origin Pro.

\section{MEASUREMENTS, RESULTS, AND DISCUSSION}

With a novel polymer-based SERS sensor, we have to first validate the function of the sensor. To see if the recorded signal is surface enhanced, we began the validation by comparing the SERS signals of the R6G sample on top of the patterned SERS structure and the smooth gold coating. The used integration time for the SERS signal recording was 15 seconds. The chip was filled with DI water to gain the reference Raman spectrum caused by the polyolefin lid. Water was replaced by a $1 \mathrm{mM}$ R6G sample and the Raman spectra were detected with $2 * 3$ point image mapping on top of the patterned SERS detection area and the smooth gold area without patterning. 


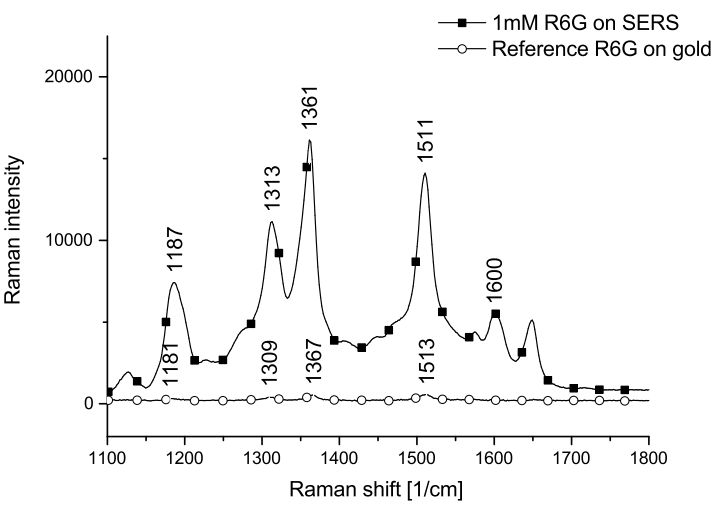

(a)

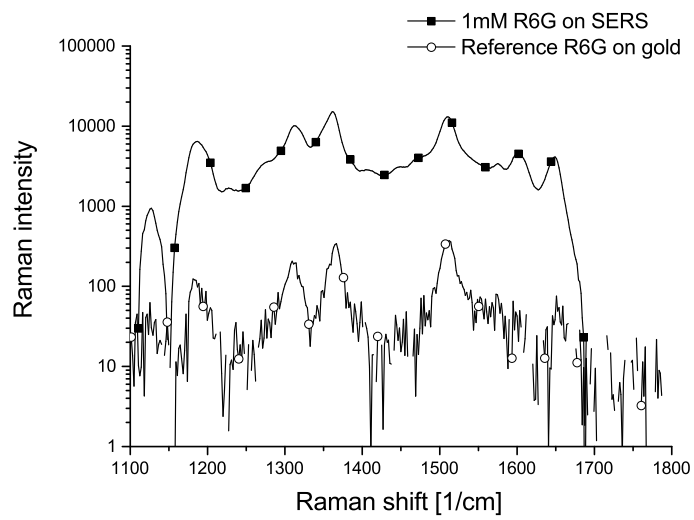

(b)

FIC. 5 a) The Raman spectra for a $1 \mathrm{mM}$ R6G solution on top of the SERS patterned area and the smooth gold area; b) The difference in the intensity can be estimated on a logarithmic scale.

The Raman spectra with subtracted background spectrum can be seen in Figure 5(a) and 5(b) with a linear and logarithmic $\mathrm{y}$-axis scale. Since the distinctive peaks for R6G are found in the Raman shift area of $11001 / \mathrm{cm}$ to $18001 / \mathrm{cm}$, this range has been used in the spectrum analysis. The results showed high R6G peaks for the patterned SERS area in comparison to the smooth gold. The peak height difference is more than 30 folds. The result can be compared to the result of Liu et al. [18], with low intensity R6G peaks for smooth a Ag/PDMS structure. As Liu states, the metal coating alone can enhance the Raman signal, although with less intensity. The results in Figure 5 and the background knowledge we have from the study of Oo et al. [4] suggests that the detected signal could be SERS originated.

To confirm the prior analysis of the SERS, and to see the effect of the optical focus on the detected signals, we conducted a trial in which we changed the focus depth of the detection. The focus was misaligned by lowering the chip to see if the R6G signal remains constant as the signal is collected from the bulk sample above the SERS surface. If the signal is generated by the non-enhanced Raman from bulk R6G in DI water solutions, the signal intensity should remain constant without varying along the change of focus depth. As we can see from the results in Figure 6, the signal intensity drops as the chip is lowered (focus level raised from the SERS surface), and thus we can, together with the observations shown in Figure 5, confirm that we are detecting surface-enhanced Raman instead of conventional Raman.

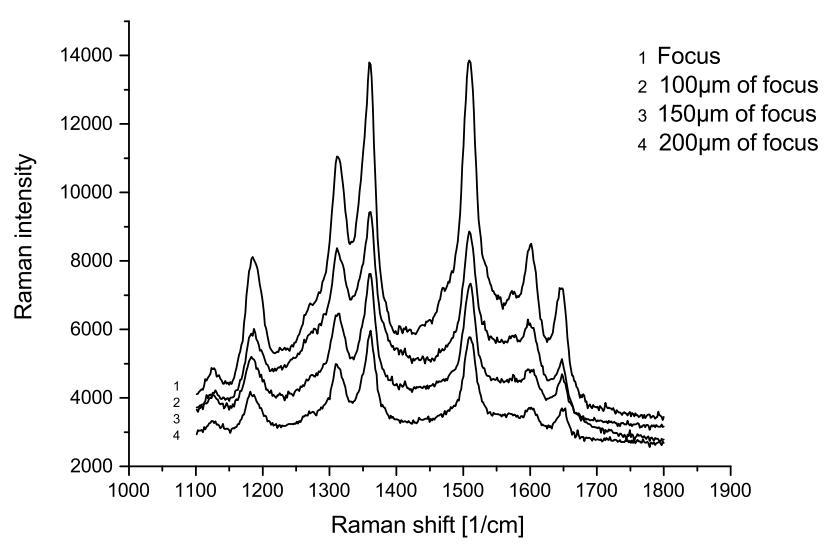

FIG. 6 Misalignment of the focus of the Raman microscope from SERS surface.

To investigate the effect of the polyolefin lid on the SERS response, we measured the R6G spectra with $10 \mu \mathrm{M}$ and $100 \mu \mathrm{M}$ concentrations. The used integration time for the SERS signal recording was 30 seconds. The chip was filled with DI water to gain the reference polyolefin spectrum. Water was replaced by R6G samples and the Raman spectra were detected. Figure 7(a) shows the Raman spectra of the R6G samples and the polyolefin reference, and Figure $7(\mathrm{~b})$ shows the $10 \mu \mathrm{M}$ and $100 \mu \mathrm{M}$ R6G spectra with subtracted polyolefin reference. The results show that although the polyolefin lid induces low Raman peaks, it has a minor effect on the R6G spectra. The effect of the lid can be further minimised by subtracting the reference spectrum from the R6G spectra.

The effect of flow dynamics on the optical SERS signal was studied with a continuous flow with $0.5 \mathrm{mM}$ R6G solution in DI $\mathrm{H}_{2} \mathrm{O}$. A similar study has been conducted previously by Hüttner et al. with a glass slide-based optofluidic SERS chip using R6G molecules in ethanol with preceding and following pure ethanol cycles [41]. In our experiment, we focused more on the dynamics of the optical signal response to the used flow velocity than on the relation of sample concentration to the signal intensity, as Hüttner et al. have done.

In the study, the fluid flow velocity was varied from $25 \mu \mathrm{l} / \mathrm{min}$ to $1000 \mu \mathrm{l} / \mathrm{min}$. The rise of the R6G signal was measured with a Raman microscope and a fluorescence microscope, as described in the Measurement set-up, to obtain the effect of the molecule diffusion and the partial mass transport limitation, and the effect of the convective flow. An image of the R6G Raman signal growth with a $50 \mu \mathrm{l} / \mathrm{min}$ flow velocity can be seen in Figure 8. The baseline tilt of the Raman spectrum was removed from the results for the analysed Raman shift area: $11001 / \mathrm{cm}$ to $18001 / \mathrm{cm}$. Peak intensity for the main R6G peaks $(11881 / \mathrm{cm}, 13101 / \mathrm{cm}, 13601 / \mathrm{cm}, 15101 / \mathrm{cm}$ and 1600 $1 / \mathrm{cm}$ ) was counted and averaged from 5 pixels. The change in the peak intensity as a function of time was calculated. Results of the measured signals were normalised and the average signal of 5 repeated measurements was calculated.

We recorded the signal rise without the dissociation phase, because the R6G molecules did not detach from the surface by washing with the $\mathrm{H}_{2} \mathrm{O}$ flow. The binding strength of the R6G 


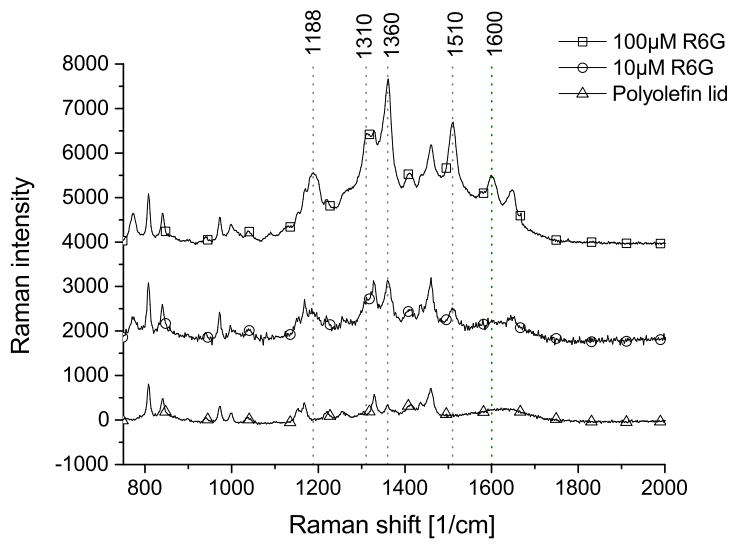

(a)

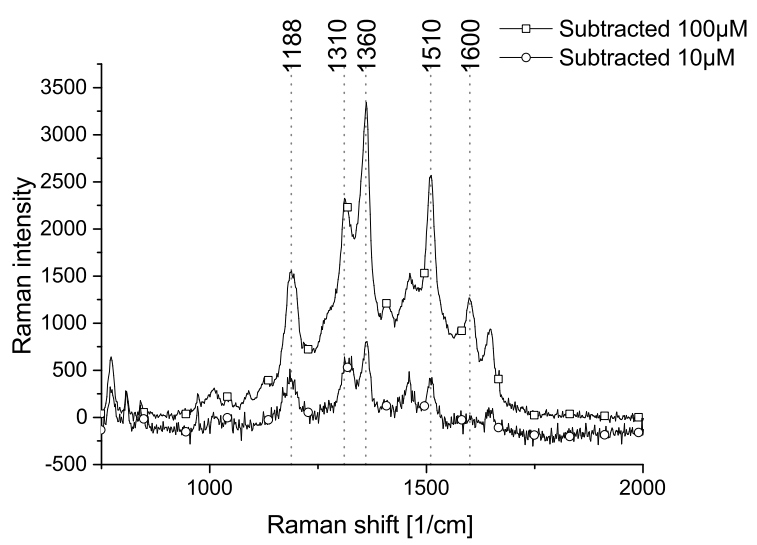

(b)

FIG. 7 a) The Raman spectra for $10 \mu \mathrm{M}$ and $100 \mu \mathrm{M}$ R6G solutions are compared to the polyolefin reference (785 $\mathrm{nm}$ laser, $40 \mathrm{~mW}$ power, $20 \mathrm{X}$ objective and $30 \mathrm{~s}$ integration time); b) The reference spectrum has been reduced from the $10 \mu \mathrm{M}$ and $100 \mu \mathrm{M}$ R6C spectra.

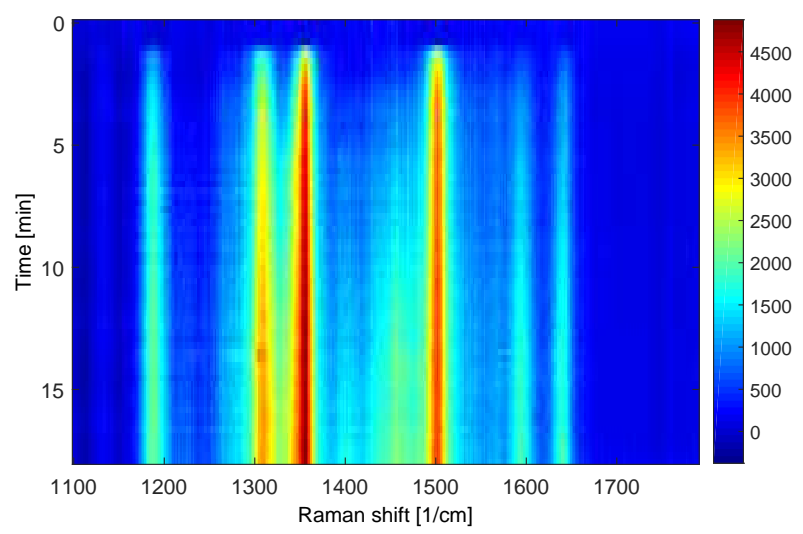

FIG. 8 Image of the signal growth during the flow trial of $0.5 \mathrm{mM} \mathrm{R6C}$ with $50 \mu \mathrm{l} / \mathrm{min}$ flow velocity.

molecules to the gold surface was too strong, and the signal did not return to zero intensity. A cleaning step was carried out by oxygen plasma etching ( 5 min $300 \mathrm{~W}$ ) between the flow runs. Each flow velocity was recorded 5 times and each chip was reused 3 times. The detected average fluorescence and SERS signals for the measured flow velocities are depicted in Figure 9(a) and 9(b) as a function of time.

To analyse the results, linear functions were fitted on the rising edge of the fluorescence and SERS signals. In Figure 9(a), the fitted functions are depicted for the fluorescence, and in

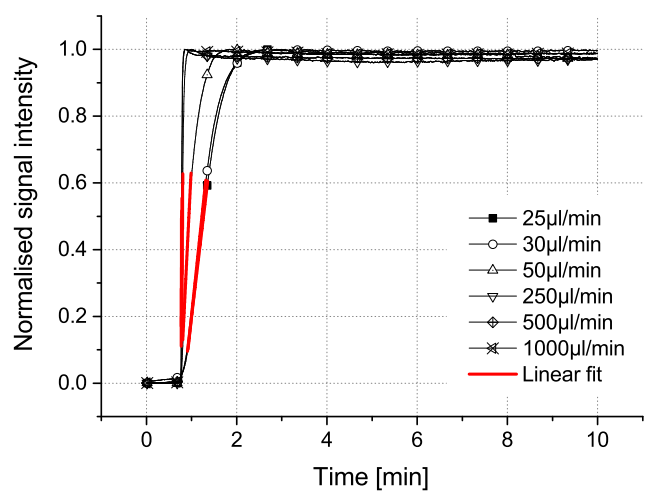

(a)

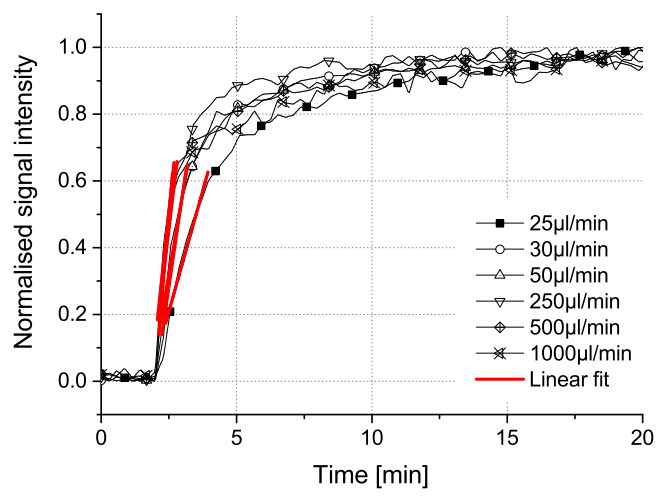

FIG. 9 a) Fluorescence signals as a function of time; b) SERS signals as a function of time. Linear function has been fitted for the rising edge of signals.

Figure 9(b) for the SERS signals. Linear functions are fitted for the range of $10 \%$ to $60 \%$ of the maximum intensity. The slope values attained are used to calculate the rise time of the signals for the aforementioned range. Figure 10(a) presents the comparative results of the SERS signal rise times and the fluorescence signal rise times. When comparing the results, it can be seen that the detected SERS signal rise is slower than the fluorescence signal rise of the R6G with all velocities in the study. The median of the lag time between the arrival of R6G molecules in the detection chamber by convective flow and the arrival and binding of R6G molecules on the SERS surface is 40.7 seconds, as depicted in Figure 10(b).

From the results, we can see that the lag time is larger for slower flow velocities. This could be due to insufficient molecule transport to the diffusion boundary layer. The lag time settles for the higher velocities and the dynamics of the diffusion and surface binding turn constant. In the future, these results will help us to plan studies with bioanalyte samples and active ligands on the surface, through the knowledge of the influence of an increasing mass transport limitation with flow velocities of $50 \mu \mathrm{l} / \mathrm{min}$ and less.

\section{CONCLUSIONS}

A novel method for fabricating a polymer-based optofluidic sensor with roll-to-roll imprinted SERS patterning and integrated adhesive microfluidic circuit was presented. The SERS substrates showed 30 folds higher enhancement than 


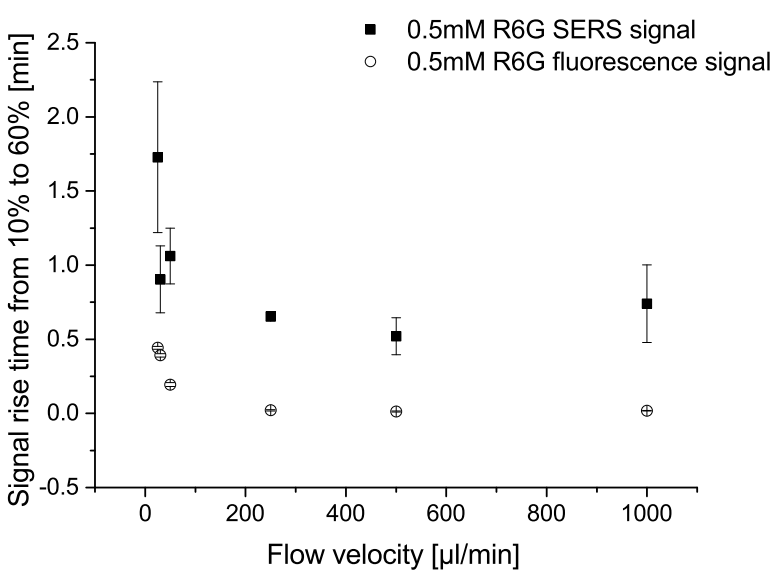

(a)

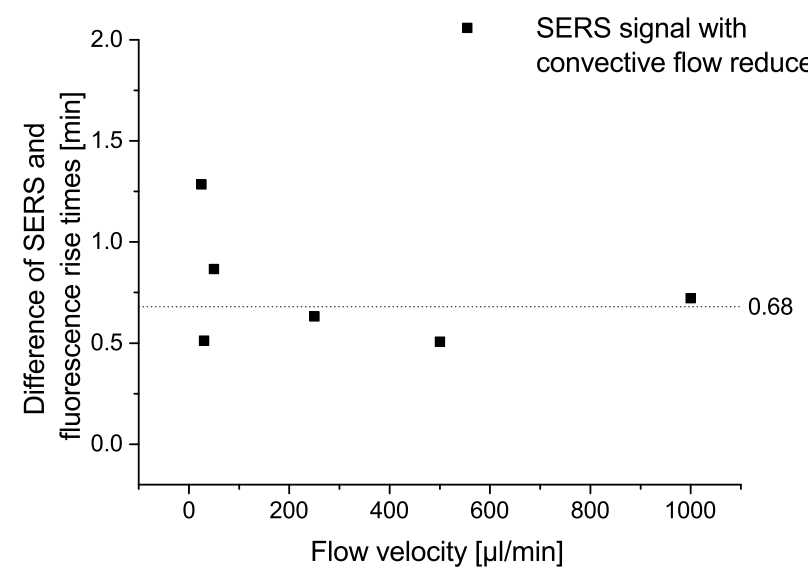

(b)

FIG. 10 a) Signal rise times for the $0.5 \mathrm{mM}$ R6C SERS signal and the fluorescence signal; b) Difference in the signal rise times between the SERS and the fluorescence signal.

the smooth evaporated gold with the $1 \mathrm{mM}$ Rhodamine 6G sample. Substrates were easily integrated into adhesive-based microfluidic devices, and the effect of the polyolefin lid could be removed from the R6G spectra. The SERS measurements of R6G fluid in the microfluidic circuit under constant flow conditions were successfully performed with a standard deviation even as low as $5 \%$ for a $250 \mu \mathrm{l} / \mathrm{min}$ flow velocity. The comparison of the signal rise of excited fluorescence with a 0.5 mM R6G sample and the recorded SERS signal showed that there is a median lag time of 40.7 seconds between the arrival of the sample molecules with the bulk flow into the detection chamber and the arrival and adsorption of the molecules on the detection surface. For flow values of $50 \mu \mathrm{l} / \mathrm{min}$ and less, the concentration of R6G molecules near the sensor surface rises slowly, and the transport is insufficient due to the depletion of analytes near the sensor surface. The high throughput fabrication can be developed in future by transferring the fabrication of the microfluidics from table top cutter to roll-to-roll process [42]. By further applying automated assembly of fluidic and optical layers instead on manual lamination, it is possible to achieve a truly disposable sensor chips offering a low cost solution for analytical applications.

\section{ACKNOWLEDGEMENTS}

This work was supported by University of Oulu Graduate School, Infotech Oulu Doctoral Programme and Tekes (Finnish Funding Agency for Innovation) under the FMA project.

\section{References}

[1] A. Liu, H. Huang, L. Chin, Y. Yu, and X. Li, "Label-free detection with micro optical fluidic systems (MOFS): a review," Anal. Bioanal. Chem. 391, 2443-2452 (2008).

[2] H. K. Hunt, and A. M. Armani, "Label-free biological and chemical sensors," Nanoscale 2, 1544-1559 (2010).

[3] M. Nirschl, F. Reuter, and J. Vörös, "Review of transducer principles for label-free biomolecular interaction analysis," Biosensors 1, 70-92 (2011).

[4] S. Z. 00, R. Chen, S. Siitonen, V. Kontturi, D. Eustace, J. Tuominen, S. Aikio, and M. Charlton, "Disposable plasmonic plastic SERS sensor," Opt. Express 21, 18484-18491 (2013).

[5] X. Fan, and I. M. White, "Optofluidic microsystems for chemical and biological analysis," Nat. Photonics 5, 591-597 (2011).

[6] K. Kneipp, Y. Wang, H. Kneipp, L. T. Perelman, I. Itzkan, R. R. Dasari, and M. S. Feld, "Single molecule detection using surface-enhanced Raman scattering (SERS)," Phys. Rev. Lett. 78, 1667 (1997).

[7] A. M. Michaels, M. Nirmal, and L. Brus, "Surface enhanced Raman spectroscopy of individual rhodamine 6C molecules on large $\mathrm{Ag}$ nanocrystals," J. Am. Chem. Soc. 121, 9932-9939 (1999).

[8] S. Nie, and S. R. Emory, "Probing Single Molecules and Single Nanoparticles by Surface-Enhanced Raman Scattering," Science 275, 1102-1106 (1997).

[9] A. Virga, P. Rivolo, F. Frascella, A. Angelini, E. Descrovi, F. Geobaldo, and F. Giorgis, "Silver nanoparticles on porous silicon: approaching single molecule detection in resonant SERS regime," J. Phys. Chem. C 117, 20139-20145 (2013).

[10] G. C. Schatz, M. A. Young, and R. P. Van Duyne, "Electromagnetic mechanism of SERS," Top. Appl. Phys. 103, 19-45 (2006).

[11] K. C. Bantz, A. F. Meyer, N. J. Wittenberg, H. Im, 0̈. Kurtulus, S. H. Lee, N. C. Lindquist, S. Oh, et al., "Recent progress in SERS biosensing," Phys. Chem. Chem. Phys. 13, 11551-11567 (2011).

[12] C. L. Haynes, and R. P. Van Duyne, "Plasmon-Sampled SurfaceEnhanced Raman Excitation Spectroscopy," J. Phys. Chem. B 107, 7426-7433 (2003).

[13] T. R. Jensen, R. P. V. Duyne, S. A. Johnson, and V. A. Maroni, "Surface-Enhanced Infrared Spectroscopy: A Comparison of Metal Island Films with Discrete and Nondiscrete Surface Plasmons," Appl. Spectrosc. 54, 371-377 (2009).

[14] L. A. Dick, A. J. Haes, and R. P. Van Duyne, "Distance and orientation dependence of heterogeneous electron transfer: a surface-enhanced resonance Raman scattering study of cytochrome $\mathrm{c}$ bound to carboxylic acid terminated alkanethiols adsorbed on silver electrodes," J. Phys. Chem. B 104, 11752-11762 (2000).

[15] M. Litorja, C. L. Haynes, A. J. Haes, T. R. Jensen, and R. P. Van Duyne, "Surface-enhanced Raman scattering detected temperature programmed desorption: optical properties, nanostructure, and stability of silver film over $\mathrm{SiO} 2$ nanosphere surfaces," J. Phys. Chem. B 105, 6907-6915 (2001). 
[16] L. A. Dick, A. D. McFarland, C. L. Haynes, and R. P. Van Duyne, "Metal film over nanosphere (MFON) electrodes for surfaceenhanced Raman spectroscopy (SERS): Improvements in surface nanostructure stability and suppression of irreversible loss," J. Phys. Chem. B 106, 853-860 (2002).

[17] I. M. White, S. H. Yazdi, and W. Y. Wei, "Optofluidic SERS: synergizing photonics and microfluidics for chemical and biological analysis," Microfluid. Nanofluid. 13, 205-216 (2012).

[18] G. L. Liu, and L. P. Lee, "Nanowell surface enhanced Raman scattering arrays fabricated by soft-lithography for label-free biomolecular detections in integrated microfluidics," Appl. Phys. Lett. 87, 074101 (2005).

[19] A. Lamberti, A. Virga, A. Angelini, A. Ricci, E. Descrovi, M. Cocuzza, and F. Giorgis, "Metal-elastomer nanostructures for tunable SERS and easy microfluidic integration," RSC Advances 5, 4404-4410 (2015).

[20] J. Teng, J. Chu, C. Liu, T. Xu, Y. Lien, J. Cheng, S. Huang, et al., "Fluid Dynamics in Microchannels," in Fluid Dynamics, Computational Modeling and Applications L.H. Juarez, ed., 403-436 (InTech, Rijeka, 2012).

[21] M. Zimmermann, E. Delamarche, M. Wolf, and P. Hunziker, "Modeling and optimization of high-sensitivity, low-volume microfluidic-based surface immunoassays," Biomed. Microdevices 7, 99-110 (2005).

[22] N. Orgovan, D. Patko, C. Hos, S. Kurunczi, B. Szabo, J. J. Ramsden, and R. Horvath, "Sample handling in surface sensitive chemical and biological sensing: A practical review of basic fluidics and analyte transport," Adv. Colloid Interfac. 211, 1-16 (2014).

[23] J. Koplik, J. R. Banavar, and J. F. Willemsen, "Molecular dynamics of fluid flow at solid surfaces," Phys. Fluids A - Fluid. 1, 781-794 (1989).

[24] J. Lauri, M. Wang, M. Kinnunen, and R. Myllylä, "Measurement of microfluidic flow velocity profile with two Doppler optical coherence tomography systems," in Biomed. Optics 2008 68630F68630F-8 (2008).

[25] J. Lauri, J. Czajkowski, R. Myllylä, and T. Fabritius, "Measuring flow dynamics in a microfluidic chip using optical coherence tomography with $1 \mu \mathrm{m}$ axial resolution," Flow Meas. Instrum. 43, 1-5 (2015).

[26] M. L. Yarmush, D. B. Patankar, and D. M. Yarmush, "An analysis of transport resistances in the operation of BIAcoreấ̌ć; implications for kinetic studies of biospecific interactions," Mol. Immunol. 33, 1203-1214 (1996).

[27] M. Stenberg, and H. Nygren, "Kinetics of antigen-antibody reactions at solid-liquid interfaces," J. Immunol. Methods 113, 3-15 (1988).

[28] T. E. Starr, and N. L. Thompson, "Total internal reflection with fluorescence correlation spectroscopy: combined surface reaction and solution diffusion," Biophys. J. 80, 1575-1584 (2001).

[29] T. Gervais, and K. F. Jensen, "Mass transport and surface reactions in microfluidic systems," Chem. Eng. Sci., 61, 1102-1121 (2006).
[30] A. Lionello, J. Josserand, H. Jensen, and H. H. Girault, "Dynamic protein adsorption in microchannels by "stop-flow" and continuous flow," Lab Chip 5, 1096-1103 (2005).

[31] G. Hu, Y. Gao, and D. Li, "Modeling micropatterned antigenantibody binding kinetics in a microfluidic chip," Biosens. Bioelectron. 22, 1403-1409 (2007).

[32] D. G. Myszka, T. A. Morton, M. L. Doyle, and I. M. Chaiken, "Kinetic analysis of a protein antigen-antibody interaction limited by mass transport on an optical biosensor," Biophys. Chem. 64, 127-137 (1997).

[33] K. Lebedev, S. Mafe, and P. Stroeve, "Convection, diffusion and reaction in a surface-based biosensor: modeling of cooperativity and binding site competition on the surface and in the hydrogel," J. Colloid Interface Sci. 296, 527-537 (2006).

[34] R. Karlsson, A. Michaelsson, and L. Mattsson, "Kinetic analysis of monoclonal antibody-antigen interactions with a new biosensor based analytical system," J. Immunol. Methods 145, 229-240 (1991).

[35] R. W. Glaser, "Antigen-antibody binding and mass transport by convection and diffusion to a surface: a two-dimensional computer model of binding and dissociation kinetics," Anal. Biochem. 213, 152-161 (1993).

[36] L. L. Christensen, "Theoretical analysis of protein concentration determination using biosensor technology under conditions of partial mass transport limitation," Anal. Biochem. 249, 153-164 (1997).

[37] R. W. Glaser, "Antigen-antibody binding and mass transport by convection and diffusion to a surface: a two-dimensional computer model of binding and dissociation kinetics," Anal. Biochem. 213, 152-161 (1993).

[38] P. M. Richalet-Secordel, N. Rauffer-Bruyere, L. L. Christensen, B. Ofenloch-Haehnle, C. Seidel, and M. H. Van Regenmortel, "Concentration measurement of unpurified proteins using biosensor technology under conditions of partial mass transport limitation," Anal. Biochem. 249, 165-173 (1997).

[39] L. Lee, "Adsorption: the solid-fluid interface," in Molecular Thermodynamics of Nonideal Fluids H. Brenner, ed., 424-462 (Butterworth-Heinemann, Boston, 1988).

[40] D. Murzin, "Physisorption and chemisorption," in Engineering Catalysis, 16 (De Gruyter, Berlin, 2013).

[41] W. Hüttner, K. Christou, A. Cöhmann, V. Beushausen, and H. Wackerbarth, "Implementation of substrates for surfaceenhanced Raman spectroscopy for continuous analysis in an optofluidic device," Microfluid. Nanofluid. 12, 521-527 (2012).

[42] R. Liedert, L. K. Amundsen, A. Hokkanen, M. Mäki, A. Aittakorpi, M. Pakanen, J. R. Scherer, et al., "Disposable roll-to-roll hot embossed electrophoresis chip for detection of antibiotic resistance gene mecA in bacteria," Lab Chip 12, 333-339 (2012). 